International Journal of Pure and Applied Mathematics

Volume 90 No. 4 2014, 561-583

ISSN: 1311-8080 (printed version); ISSN: 1314-3395 (on-line version)

url: http://www.ijpam.eu

doi: http://dx.doi.org/10.12732/ijpam.v90i4.14

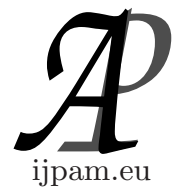

\title{
MULTILINEAR STIELTJES INTEGRALS OF FUNCTIONS OF SEVERAL VARIABLES
}

\author{
A. Halilović ${ }^{1} \S$, J. Panrike ${ }^{2}$ \\ ${ }^{1,2}$ The Royal Institute of Technology \\ KTH STH, Campus Haninge \\ Marinens v. 30, 13640 Haninge, SWEDEN
}

\begin{abstract}
In this paper we define and consider multilinear Stieltjes integrals for vector-valued functions defined on a closed bounded interval in $\mathbb{R}^{n}$. The convergence of the Stieltjes sums is considered in the Riemann, Moore-Pollard and Henstock-Kurzweil sense.
\end{abstract}

AMS Subject Classification: 46G10, 28B05

Key Words: multilinear integral, Stieltjes integral, Henstock-Kurzweil integral, vector-valued functions, several variables

\section{Introduction}

Multilinear integrals are Stieltjes-type integrals in which the number of integrands or the number of integrators (or both) can be greater than one, e.g. $\int_{B} \mathcal{A}\left(f_{1}, d g_{1}, f_{2}\right)$ or $\int_{B} \mathcal{A}\left(f_{1}, d g_{1}, f_{2}, d g_{2}\right)$. Multilinear integrals for vectorvalued functions of one real variable have already been defined and studied by A Halilović, see for ex. [2]-[4]. In this article we expand the definition of the multilinear Stieltjes integral to $n$-dimensional space. The integrands

Received: January 20, 2014

(C) 2014 Academic Publications, Ltd. url: www.acadpubl.eu

${ }^{\S}$ Correspondence author 
$f_{k}: B \rightarrow X_{k}, k=1, \ldots, p$ and the integrators $g_{l}: B \rightarrow Y_{l}, l=1, \ldots, q$ are functions defined on a closed bounded interval $B \subset \mathbb{R}^{n}$ with values in linear normed spaces $X_{k}, k=1, \ldots, p, Y_{l}, l=1, \ldots, q$. To simplify the notation we consider multilinear integrals in the case when the integrands $f_{k}$ are placed in the beginning and integrators $g_{k}$ at the end of the expression $\mathcal{A}\left(f_{1}, \ldots, f_{p}, d g_{1}, \ldots, d g_{q}\right)$, but all the results are valid for any order of integrands and integrators. First we define Stieltjes quasi volume and then, using a bounded multilinear operator $\mathcal{A}: X_{1} \times \cdots \times X_{p} \times Y_{1} \cdots \times Y_{q} \rightarrow Z$, where $Z$ is a Banach space, we define the multilinear integral

$$
\int_{B} \mathcal{A}\left(f_{1}, \ldots, f_{p}, d g_{1}, \ldots, d g_{q}\right)
$$

as the limit of Stieltjes sums. Stieltjes sums and the limiting process can be defined in different ways and this leads to different types of integrals. In this article we consider Riemann, Moore-Pollard and Henstock-Kurzweil multilinear integrals and give some basic properties of these integrals. Two existence theorems are given:

In the first, referred to here as Theorem 4.1, we prove the existence of the Riemann-Stieltjes multilinear integral assuming that:

1. The integrands $f_{k}$ are continuous functions.

2. Stieltjes quasi volumes are of bounded semivariations.

3. The expression $\mathcal{A}\left[f_{1}\left(s_{1}\right), \ldots, f_{p}\left(s_{p}\right), \sigma_{g_{1}}(C), \ldots, \sigma_{g_{q}}(C)\right]$ is interval additive.

In the second, referred to here as Theorem 5.1, we prove that the existence of the Moore-Pollard multilinear integral together with the interval additivity of $\mathcal{A}\left[f_{1}\left(s_{1}\right), \ldots, f_{p}\left(s_{p}\right), \sigma_{g_{1}}(C), \ldots, \sigma_{g_{q}}(C)\right]$ implies the existence of the HenstockKurzweil multilinear integral.

\section{Definitions and Terminology}

We begin with some definitions and terminology that are used throughout this article.

Definition 2.1. By an n-dimensional block (or n-dimensional closed interval), or just block, we mean a set of points

$$
B=\left\{\left(x_{1}, \ldots, x_{n}\right): a_{1} \leq x_{1} \leq b_{1}, \ldots, a_{n} \leq x_{n} \leq b_{n}\right\},
$$


where $a_{1} \leq b_{1}, \ldots, a_{n} \leq b_{n}$. We shall also occasionally write $B=[a, b]$ where $a=\left(a_{1}, \ldots, a_{n}\right)$ and $b=\left(b_{1}, \ldots, b_{n}\right)$.

The volume of $B$ is

$$
v(B)=\prod_{i=1}^{n}\left(b_{i}-a_{i}\right)
$$

Any point $\gamma=\left(c_{1}, \ldots, c_{n}\right)$, where $c_{k}=a_{n}$ or $c_{k}=b_{n}$, is called a vertex of $B$. Note that $B$ has $2^{n}$ vertices.

Any set

$$
\Lambda_{k}=\left\{\left(x_{1}, \ldots, x_{n}\right): a_{1} \leq x_{1} \leq b_{1}, \ldots, x_{k}=c_{k}, \ldots, a_{n} \leq x_{n} \leq b_{n}\right\},
$$

where $c_{k}=a_{k}$ or $c_{k}=b_{k}$ will be called a face of the block $B$.

The interior of $B$ we denote by $\operatorname{int}(B)$.

The number $|B|=\max \left\{b_{1}-a_{1}, \ldots, b_{n}-a_{n}\right\}$ will be called the size of the block $B$.

A block $B^{\prime}$ is a subblock of a block $B$ if $B^{\prime} \subseteq B$.

Definition 2.2. We say that $\Pi=\left\{B_{1}, \ldots, B_{r}\right\}$, consisting of a finite family of subblocks of $B$, forms a partition of $B$ if $B=\cup B_{k}, k=1, \ldots, r$ and $i \neq j \Rightarrow \operatorname{int}\left(B_{i}\right) \cap \operatorname{int}\left(B_{j}\right)=\varnothing$.

The number

$$
|\Pi|=\max \left\{\left|B_{k}\right|\right\}
$$

is called the norm (or mesh) of the partition $\Pi$.

Definition 2.3. If a partition $\Pi^{\prime}$ is obtained by further subdividing the blocks of the partition $\Pi$ we say that $\Pi^{\prime}$ is a refinement of $\Pi$.

Definition 2.4. (Stieltjes quasi volume.) Let $B=[a, b]$ with $a=$ $\left(a_{1}, \ldots, a_{n}\right)$ and $b=\left(b_{1}, \ldots, b_{n}\right)$. Let $g: B \rightarrow X$ be a vector-valued function defined on $B$ with values in a Banach space $X$. Let $\gamma=\left(c_{1}, \ldots, c_{n}\right)$ where $c_{k}=a_{n}$ or $c_{k}=b_{n}$ and $\nu(\gamma)$ denotes the number of terms in $\gamma$ for which $c_{k}=a_{n}$. We define, in a common way (see for example [7] or [6]) a Stieltjes quasi volume $\sigma_{g}(B)$ of the block $B$ associated to the function $g$ by

$$
\sigma_{g}(B)=\sum_{\gamma}(-1)^{\nu(\gamma)} g(\gamma)
$$

where the summation is over all vertices $\gamma=\left(c_{1}, \ldots, c_{n}\right)$ where $c_{k}=a_{n}$ or $c_{k}=b_{n}$, of the block $B$. 
For example, when $n=3$,

$$
\begin{gathered}
\sigma_{g}(B)=g\left(b_{1}, b_{2}, b_{3}\right)+g\left(a_{1}, a_{2}, b_{3}\right)+g\left(a_{1}, b_{2}, a_{3}\right)+g\left(b_{1}, a_{2}, a_{3}\right) \\
-g\left(a_{1}, b_{2}, b_{3}\right)-g\left(b_{1}, a_{2}, b_{3}\right)-g\left(b_{1}, b_{2}, a_{3}\right)-g\left(a_{1}, a_{2}, a_{3}\right)
\end{gathered}
$$

Note that Stieltjes quasi volume is a generalization of the ordinary volume $v(B)$ of a block B. For example, we can compare $\sigma_{g}(B)$ in the above example with the volume of $B$ :

$$
\begin{gathered}
v(B)=\prod_{i=1}^{3}\left(b_{i}-a_{i}\right) \\
=b_{1} b_{2} b_{3}+a_{1} a_{2} b_{3}+a_{1} b_{2} a_{3}+b_{1} a_{2} a_{3}-a_{1} b_{2} b_{3}-b_{1} a_{2} b_{3}-b_{1} b_{2} a_{3}-a_{1} a_{2} a_{3} .
\end{gathered}
$$

Definition 2.5. A vector-valued function of blocks $F(C)$ is intervaladditive if for any partition of $C$ consisting of the blocks $C_{k}, k=1, \ldots, r$ we have $F(C)=\sum_{k=1}^{r} F\left(C_{k}\right)$.

Remark 2.1. Using the operator

$$
\Delta_{k}^{b_{k}} b_{k} g\left(x_{1}, \ldots, x_{n}\right)=g\left(x_{1}, \ldots, b_{k}, \ldots, x_{n}\right)-g\left(x_{1}, \ldots, a_{k}, \ldots, x_{n}\right)
$$

we can express Stieltjes quasi volume $\sigma_{g}(B)$ of the block $B$ associated to the function $g$ as

$$
\sigma_{g}(B)=\Delta_{n a_{n}}^{b_{n}} \cdots \Delta_{2}^{b_{a_{2}}} \Delta_{1}^{b_{a_{1}}} g\left(x_{1}, \ldots, x_{n}\right) .
$$

For example, in two dimensions

$$
\begin{aligned}
\sigma_{g}(B) & =\Delta_{2}{ }_{a_{2}}^{b_{2}} \Delta_{1}{ }_{a_{1}}^{b_{1}} g\left(x_{1}, x_{2}\right)=\Delta_{2} a_{2} b_{2} \\
& \left.=g\left(b_{1}, b_{2}\right)-g\left(b_{1}, a_{2}\right)-g\left(a_{1}, b_{2}\right)+g\left(a_{1}, x_{2}\right)\right]
\end{aligned}
$$

Then it is easy to prove that $\sigma_{g}(B)$ is an interval-additive function.

Remark 2.2. The following properties follow from the definition of the Stieltjes quasi volume:

1. If $B$ is a degenerated interval, that is $a_{k}=b_{k}$ for some $k$, then $\sigma_{g}(B)=0$.

2. If $h=f+g$ then $\sigma_{h}(B)=\sigma_{f}(B)+\sigma_{g}(B)$.

3. If $h=k g$, where $\mathrm{k}$ is a real or complex number, then $\sigma_{h}(B)=k \sigma_{g}(B)$. 


\section{Riemann-Stieltjes Multilinear Integral in $\mathbb{R}^{n}$}

\section{Stieltjes sums}

Let $X_{k}, k=1, \ldots, p, Y_{l}, l=1, \ldots, q$ and $Z$ be linear normed spaces over the same field $\mathbb{R}$ or $\mathbb{C}$. Let $Z$ be a Banach space. Let

$$
\mathcal{A}: X_{1} \times \cdots \times X_{p} \times Y_{1} \cdots \times Y_{q} \rightarrow Z
$$

be a bounded multilinear operator and let

$$
\begin{gathered}
f_{k}: B \rightarrow X_{k}, k=1, \ldots, p, \\
g_{l}: B \rightarrow Y_{l}, l=1, \ldots, q
\end{gathered}
$$

be vector-valued functions defined on a block $B$.

We define two types of Stieltjes sums, I and II, which differ in number of associated points in each subblock.

Type I. Let $\Pi=\left\{B_{1}, \ldots, B_{r}\right\}$ be a partition of $B$. We arbitrarily choose $p$ points $s_{1}^{i}, \ldots, s_{p}^{i}$ in each block $B_{i}$ which we substitute into $f_{1}, \ldots, f_{p}$, and form the Stieltjes sum of the first type

$$
S_{I}(\Pi)=\sum_{i=1}^{r} \mathcal{A}\left[f_{1}\left(s_{1}^{i}\right), \ldots, f_{p}\left(s_{p}^{i}\right), \sigma_{g_{1}}\left(B_{i}\right), \ldots, \sigma_{g_{q}}\left(B_{i}\right)\right] .
$$

Type II. We choose one point $s_{i}$ in each block $B_{i}$ and form the Stieltjes sum of the second type

$$
S_{I I}(\Pi)=\sum_{i=1}^{r} \mathcal{A}\left[f_{1}\left(s_{i}\right), \ldots, f_{p}\left(s_{i}\right), \sigma_{g_{1}}\left(B_{i}\right), \ldots, \sigma_{g_{q}}\left(B_{i}\right)\right]
$$

In the above sums $\sigma_{g_{k}}\left(B_{i}\right)$ denotes quasi volume of the block $B_{i}$ associated to the function $g_{k}$.

Definition 3.1. We say that the type I multilinear Riemann-Stieltjes integral of $f_{1}, \ldots, f_{p}$ with respect to $\mathcal{A}$ and $g_{1}, \ldots, g_{q}$, exists on $B$ if there exists a vector $J_{I} \in Z$ with the following property:

For every $\epsilon>0$, there is a constant $\delta>0$, such that for any partition $\Pi=\left\{B_{1}, \ldots, B_{r}\right\}$ of the block $B$, and any choice of points $s_{1}^{i}, \ldots, s_{p}^{i}$ in $B_{i}$, $i=1, \ldots, r$ we have

$$
|\Pi|<\delta \Rightarrow\left|J_{I}-S_{I}(\Pi)\right|<\epsilon
$$


Then we write

$$
J_{I}=\left(R S_{I}\right) \int_{B} \mathcal{A}\left(f_{1}, \ldots, f_{p}, d g_{1}, \ldots, d g_{q}\right) .
$$

We shall also occasionally write

$$
J_{I}=\left(R S_{I}\right) \int_{B} \mathcal{A}(F, d G)
$$

and here $F$ and $d G$ stand for $f_{1}, \ldots, f_{p}$ and $d g_{1}, \ldots, d g_{q}$ respectively but this has the exact same meaning as the previous expression for $J_{I}$. Thus

$$
\left(R S_{I}\right) \int_{B} \mathcal{A}(F, d G)
$$

is not a bilinear integral; it is merely a less cumbersome notation for the multilinear integral.

Definition 3.2. By changing type I Stieltjes sums to type II sums in the above definition we get the definition of type II multilinear Riemann-Stieltjes integral

$$
J_{I I}=\left(R S_{I I}\right) \int_{B} \mathcal{A}\left(f_{1}, \ldots, f_{p}, d g_{1}, \ldots, d g_{q}\right) .
$$

Remark 3.1. It is obvious that the existence of the type I integral implies the existence of the type II integral.

Remark 3.2. If we do not mention the type of a Stieltjes integral then, in this article, we mean that the integral we consider is of type I.

Using the above definitions and the fact that $Z$ is a Banach space it is easy to prove the following lemmas.

Lemma 3.1. If the integral $\left(R S_{I}\right) \int_{B} \mathcal{A}(F, d G)$ exists then it is uniquely determined.

Lemma 3.2. (Cauchy condition I) The integral

$$
\left(R S_{I}\right) \int_{B} \mathcal{A}(F, d G)
$$

exists if and only if for every $\epsilon>0$ there exists a constant $\delta>0$, such that for any two partitions $\Pi_{1}$ and $\Pi_{2}$ satisfying $\left|\Pi_{1}\right|<\delta$ and $\left|\Pi_{2}\right|<\delta$ we have

$$
\left|S_{I}\left(\Pi_{1}\right)-S_{I}\left(\Pi_{2}\right)\right|<\epsilon
$$


Lemma 3.3. (Cauchy condition II) The integral

$$
\left(R S_{I}\right) \int_{B} \mathcal{A}(F, d G)
$$

exists if and only if for every $\epsilon>0$ there exists a constant $\delta>0$, such that for any partition $\Pi_{1}$ satisfying $\left|\Pi_{1}\right|<\delta$ and its (arbitrary) refinement $\Pi_{2}$ we have

$$
\left|S_{I}\left(\Pi_{1}\right)-S_{I}\left(\Pi_{2}\right)\right|<\epsilon
$$

\section{Henstock-Kurzweil-Stieltjes Multilinear Integrals}

The difference between Riemann-type integration and Henstock-Kurzweil (HK) integration is that $\delta$ in the definition of HK-integrals is not necessarily a constant but a positive function defined on $B$, that is

$$
\delta: B \rightarrow \mathbb{R}_{+} \quad\left(\text { where } \mathbb{R}_{+}=(0, \infty)\right) .
$$

The function $\delta$ will be called a gauge on $B$. The basic concept in the theory of the HK integral is that of a tagged $\delta$-fine partition; see [1], [5] or [6]. We begin by defining two types of tagged partitions which differ in number of points in each subinterval: type I with $p$ associated points and type II with only one point associated to each subinterval.

Definition 4.1. Let $B$ be an $n$-dimensional block. Let $\Pi=\left\{B_{1}, \ldots, B_{r}\right\}$ be a partition of $B$, and let

$$
D_{I}=\left\{\left(\mathfrak{S}_{i}, B_{i}\right), i=1, \ldots, r\right\}
$$

denote the collection of blocks of $\Pi$ together with the associated points $\mathfrak{S}_{i}=$ $\left\{s_{j}^{i}\right\}$, where $s_{j}^{i} \in B_{i}, j=1, \ldots, p$. We say that $D_{I}$ is a tagged partition of type I of the block $B$ and refer to the points $s_{j}^{i}$ as the tags of $D_{I}$.

Remark 4.1. A type I tagged partition has the following properties:

1. A tagged partition $D_{I}$ has $p$ tags associated to the block $B_{i}$.

2. Some (or all) tags $s_{j}^{i}, j=1, \ldots, p$ can be equal.

3. Some (or all) tags $s_{j}^{i}, j=1, \ldots, p$ can belong to the faces of the block $B_{i}$. 
Definition 4.2. A tagged partition which has (only) one point $s_{i}$ associated to each block $B_{i}$ will be denoted $D_{I I}$. Thus

$$
D_{I I}=\left\{\left(s_{i}, B_{i}\right), i=1, \ldots, r\right\} .
$$

$D_{I I}$ will be called a tagged partition of type II.

We now define $\delta$-fine partition.

Definition 4.3. Let $\delta$ be a gauge, that is $\delta: B \rightarrow \mathbb{R}_{+}$. We say that a tagged partition $D_{I}$ of the block $B$ is $\delta$-fine if for every $i$

$$
\left|B_{i}\right|<\min \left(\delta\left(s_{j}^{i}\right), j=1, \ldots, p\right) .
$$

In other words, for each block $B_{i}$ we have

$$
s_{j}^{i} \in B_{i} \text { and } \quad\left|B_{i}\right|<\delta\left(s_{j}^{i}\right), \quad \text { for all } j=1, \ldots, p .
$$

Changing $s_{j}^{i}$ to $s_{i}$ in the above definition we get the $\delta$-fine partition of type II. Thus,

$$
D_{I I}=\left\{\left(s_{i}, B_{i}\right), i=1, \ldots, r\right\}
$$

is $\delta$-fine if

$$
\left|B_{i}\right|<\delta\left(s_{i}\right), i=1, \ldots, r \text {. }
$$

When the tagged partition $D$ (type I or II) is $\delta$-fine we say that $D$ is subordinate to $\delta$ and we then write $D \ll \delta$.

Note that only tagged partitions can be $\delta$-fine. Therefore we can (and will from now on) omit the word "tagged" in the expression " $\delta$-fine tagged partition" and simply write " $\delta$-fine partition".

Remark 4.2. Let $D_{I I}=\left\{\left(s_{i}, B_{i}\right), i=1, \ldots, r\right\}$ be a $\delta$-fine partition of type II. Then simply taking $s_{1}^{i}=s_{2}^{i}=\cdots=s_{p}^{i}=s_{i}$ we get a $\delta$-fine partition of type I, $D_{I}=\left\{\left(\left\{s_{j}^{i}\right\}, B_{i}\right), i=1, \ldots, r\right\}$.

The following lemma shows the existence of $\delta$-fine partitions for any given positive function (gauge) $\delta: B \rightarrow \mathbb{R}_{+}$.

Lemma 4.1. Let $B$ be an $n$-dimensional block. For any gauge $\delta$ on $B$ there exists at least one $\delta$-fine partition $D_{I}$ of $B$.

Proof. A simple proof of the existence of a type II $\delta$-fine partition can be found in [5], page 42 . In other words, there exists at least one $\delta$-fine partition

$$
D_{I I}=\left\{\left(s_{i}, B_{i}\right), i=1, \ldots, r\right\}
$$


such that

$$
\left|B_{i}\right|<\delta\left(s_{i}\right), i=1, \ldots, r .
$$

Now, putting $s_{j}^{i}=s_{i}, j=1, \ldots, p$ we define a type I partition

$$
D_{I}=\left\{\left(\left\{s_{j}^{i}\right\}, B_{i}\right), j=1, \ldots, p \quad i=1, \ldots, r\right\}
$$

which satisfies

$$
s_{j}^{i} \in B_{i} \quad \text { and } \quad\left|B_{i}\right|<\delta\left(s_{j}^{i}\right), \quad \text { for all } j=1, \ldots, p .
$$

Thus, there exists at least one $\delta$-fine partition of type I.

\section{HKS-integral, type I}

The associated Stieltjes sum of the first type we define in the same way as in the definition of the RS-integral,

$$
S_{I}\left(D_{I}\right)=\sum_{i=1}^{r} \mathcal{A}\left[f_{1}\left(s_{1}^{i}\right), \ldots, f_{p}\left(s_{p}^{i}\right), \sigma_{g_{1}}\left(B_{i}\right), \ldots, \sigma_{g_{q}}\left(B_{i}\right)\right],
$$

and now Lemma 4.1 makes the following definition possible.

Definition 4.4. (Henstock-Kurzweil-Stieltjes multilinear integral) We say that the type I multilinear Stieltjes integral of $f_{1}, \ldots, f_{p}$ with respect to $\mathcal{A}$ and $g_{1}, \ldots, g_{q}$, exists in the Henstock-Kurzweil sense on $B$, if there exists a vector $J_{I} \in Z$ with the following property:

For every $\epsilon>0$, there is a gauge $\delta$ on $B$ such that for any $\delta$-fine partition $D_{I}$ we have

$$
\left|J_{I}-S_{I}\left(D_{I}\right)\right|<\epsilon
$$

We then write

$$
J_{I}=\left(H K S_{I}\right) \int_{B} \mathcal{A}\left(f_{1}, \ldots, f_{p}, d g_{1}, \ldots, d g_{q}\right)
$$

and occasionally we write simply $J_{I}=\left(H K S_{I}\right) \int_{B} \mathcal{A}(F, d G)$.

Definition 4.5. By changing type I Stieltjes sums to type II sums in the above definition we get the definition of type II multilinear Henstock-KurzweilStieltjes integral

$$
J_{I I}=\left(H K S_{I I}\right) \int_{B} \mathcal{A}\left(f_{1}, \ldots, f_{p}, d g_{1}, \ldots, d g_{q}\right) .
$$


Lemma 4.2. Let $D_{I}^{(1)}$ and $D_{I}^{(2)}$ be any two partitions of $B$ and assume that $D_{I}^{(1)}$ is $\delta_{1}$-fine and that $D_{I}^{(2)}$ is $\delta_{2}$-fine. Then there exists a tagged partition $D_{I}^{(3)}$ of $B$ with the following properties:

1. $D_{I}^{(3)}$ is both $\delta_{1}$-fine and $\delta_{2}$-fine

2. The set of tags of $D_{I}^{(3)}$ contains all tags of $D_{I}^{(1)}$ and $D_{I}^{(2)}$.

Proof. Let $D_{I}^{(1)}=\left\{\left(\left\{s_{j}^{i}\right\}, B_{i}\right)\right\}$ and $D_{I}^{(2)}=\left\{\left(\left\{t_{l}^{k}\right\}, C_{k}\right)\right\}$ be any two partitions of $B$ which are $\delta_{1}$-fine and $\delta_{2}$-fine respectively. Let $E=\left\{s_{j}^{i}\right\} \cup\left\{t_{l}^{k}\right\}=$ $\left\{Y_{1}, Y_{2}, \ldots, Y_{N}\right\}$ denote the finite set of all different tags in both $D_{I}^{(1)}$ and $D_{I}^{(2)}$. Let $d(P, Q)$ denote the (non-euclidian) distance

$$
d(P, Q)=\max \left\{\left|p_{k}-q_{k}\right|, k=1, \ldots, n\right\}
$$

between points $P=\left(p_{1}, \ldots, p_{n}\right)$ and $Q=\left(q_{1}, \ldots, q_{n}\right)$. Let

$$
\delta_{3}=\min \left\{d\left(Y_{k}, Y_{l}\right), k \neq l\right\}
$$

and

$$
d(P, E)=\min \left\{d\left(P, Y_{k}\right), k=1, \ldots, N\right\} .
$$

We define

$$
\delta= \begin{cases}\min \left\{\delta_{1}(P), \delta_{2}(P), \frac{1}{2} \delta_{3}\right\} & \text { if } P \in E, \\ \min \left\{\delta_{1}(P), \delta_{2}(P), \frac{1}{2} d(P, E), \frac{1}{2} \delta_{3}\right\} & \text { if } P \in B \backslash E .\end{cases}
$$

Then we have:

1. The function $\delta$ is a gauge on $B$; thus, according to Lemma 4.1, there exists a $\delta$-fine partition of $B$.

2. Since $\delta \leq \delta_{1}$ and $\delta \leq \delta_{2}$, any partition that is $\delta$-fine will also be $\delta_{1}$-fine and $\delta_{2}$-fine.

3. If $Y_{k}$ belongs to a block $B_{j}$ of a $\delta$-fine partition then $Y_{k}$ must be a tag. Thus, any $\delta$-fine partition (type I or II) will contain all tags $\left\{Y_{1}, Y_{2}, \ldots, Y_{N}\right\}$.

Lemma 4.3. If the integral $\left(H K S_{I}\right) \int_{B} A(F, d G)$ exists then it is uniquely determined. 
Proof. Assume that both $J_{I}$ and $J_{I}^{\prime}$ in $Z$ fulfill the requirements of Definition 4.4 for an arbitrary $\epsilon>0$. Denote by $\delta$ and $\delta^{\prime}$ the corresponding gauge functions. Then $\left|J_{I}-S_{I}\left(D_{I}\right)\right|<\epsilon$ for any $\delta$-fine partition $D_{I}$ and $\left|J_{I}-S_{I}\left(D_{I}\right)\right|<\epsilon$ for any $\delta^{\prime}$-fine partition $D_{I}^{\prime}$. Let $\delta^{\prime \prime}=\min \left(\delta, \delta^{\prime}\right)$ and let $D_{I}^{\prime \prime}$ be a $\delta^{\prime \prime}$-fine partition. Then $D_{I}^{\prime \prime}$ is a partition of $B$ which is both $\delta$-fine and $\delta^{\prime}$-fine. Therefore $\left|J_{I}-S_{I}\left(D_{I}^{\prime \prime}\right)\right|<\epsilon$ and $\left|J_{I}^{\prime}-S_{I}\left(D_{I}^{\prime \prime}\right)\right|<\epsilon$ giving

$$
\left|J_{I}-J_{I}^{\prime}\right| \leq\left|J_{I}-S_{I}\left(D_{I}^{\prime \prime}\right)\right|+\left|S_{I}\left(D_{I}^{\prime \prime}\right)-J_{I}^{\prime}\right|<\epsilon+\epsilon=2 \epsilon .
$$

Since $\epsilon>0$ was arbitrary we conclude that $J_{I}=J_{I}^{\prime}$.

Lemma 4.4. (Cauchy condition) The integral

$$
\left(H K S_{I}\right) \int_{B} \mathcal{A}(F, d G)
$$

exists if and only if for every $\epsilon>0$ there exists a gauge $\delta$ on $B$, such that for any $\delta$-fine partitions $D_{I}^{\prime}$ and $D_{I}^{\prime \prime}$ we have

$$
\left|S_{I}\left(D_{I}^{\prime}\right)-S_{I}\left(D_{I}^{\prime \prime}\right)\right|<\epsilon .
$$

Proof. $(\Rightarrow)$ Assume that $\left(H K S_{I}\right) \int_{B} \mathcal{A}(F, d G)$ exists and has the value $J \in$ $Z$. Let $\epsilon>0$ be arbitrary and choose a gauge function $\delta$ such that whenever a partition $D_{I}$ of $B$ is $\delta$-fine we have $\left|S_{I}\left(D_{I}\right)-J\right|<\epsilon / 2$ regardless of the choice of $D_{I}$. For arbitrary partitions $D_{I}^{(1)}$ and $D_{I}^{(2)}$ that are $\delta$-fine we then have the estimate

$$
\left|S_{I}\left(D_{I}^{\prime}\right)-S_{I}\left(D_{I}^{\prime \prime}\right)\right| \leq\left|S_{I}\left(D_{I}^{\prime}\right)-J\right|+\left|J-S_{I}\left(D_{I}^{\prime \prime}\right)\right|<\epsilon / 2+\epsilon / 2=\epsilon .
$$

$(\Leftarrow)$ Assume the converse and, for each $n=1,2, \ldots$, choose $\delta_{n}$ such that for any $\delta_{n}$-fine partitions $D_{I}^{\prime}$ and $D_{I}^{\prime \prime}$ we have

$$
\left|S_{I}\left(D_{I}^{\prime}\right)-S_{I}\left(D_{I}^{\prime \prime}\right)\right|<\frac{1}{n} .
$$

We may assume that $\delta_{n+1} \leq \delta_{n}$; otherwise we replace $\delta_{n+1}$ by

$$
\eta_{n+1}=\min \left\{\delta_{1}, \ldots, \delta_{n+1}\right\} \text {. }
$$

For each $n$, choose a $\delta_{n}$-fine partition $D_{I}^{(n)}$. Now, if $m>n$ then $\delta_{m}<\delta_{n}$ so that both $D_{I}^{(m)}$ and $D_{I}^{(n)}$ are $\delta_{n}$-fine. Therefore, if $m>n$, using (4.1) we have

$$
\left|S_{I}\left(D_{I}^{(n)}\right)-S_{I}\left(D_{I}^{(m)}\right)\right|<\frac{1}{n} .
$$


This shows that the sequence

$$
\left\{S_{I}\left(D_{I}^{(k)}\right), k=1,2, \ldots,\right\}
$$

is a Cauchy sequence in the complete space $Z$ and therefore convergent. Let

$$
J=\lim _{k \rightarrow \infty} S_{I}\left(D_{I}^{(k)}\right)
$$

Passing to the limit as $m \rightarrow \infty$ in (4.2) we get

$$
\left|S_{I}\left(D_{I}^{(n)}\right)-J\right| \leq \frac{1}{n}
$$

Given arbitrary $\epsilon>0$, let $N$ be chosen so that $\frac{1}{N}<\frac{\epsilon}{2}$. Let $D_{I}$ be an arbitrary $\delta_{N}$-fine partition. Then, using (4.1) and (4.3), we have

$$
\begin{aligned}
\left|S_{I}\left(D_{I}\right)-J\right| \leq & \left|S_{I}\left(D_{I}\right)-S_{I}\left(D_{I}^{(N)}\right)\right|+\left|S_{I}\left(D_{I}^{(N)}\right)-J\right| \\
& \leq \frac{1}{N}+\frac{1}{N}<\frac{\epsilon}{2}+\frac{\epsilon}{2}=\epsilon .
\end{aligned}
$$

This shows that $\left(H K S_{I}\right) \int_{B} \mathcal{A}(F, d G)$ exists and the proof is complete.

Here are some properties of the HKS-integral.

Lemma 4.5. a) Let $f_{i}=f_{i, a}+f_{i, b}$ for some $i, 1 \leq i \leq p$. If the integrals

$$
J_{a}=\left(H K S_{I}\right) \int_{B} \mathcal{A}\left(f_{1}, \ldots, f_{i, a}, \ldots, f_{p}, d g_{1}, \ldots, d g_{q}\right)
$$

and

$$
J_{b}=\left(H K S_{I}\right) \int_{B} \mathcal{A}\left(f_{1}, \ldots, f_{i, b}, \ldots, f_{p}, d g_{1}, \ldots, d g_{q}\right)
$$

exist then the integral

$$
J=\left(H K S_{I}\right) \int_{B} \mathcal{A}\left(f_{1}, \ldots, f_{i}, \ldots, f_{p}, d g_{1}, \ldots, d g_{q}\right)
$$

also exists and $J=J_{a}+J_{b}$.

b) Let $g_{j}=g_{j, a}+g_{j, b}$ for some $j, 1 \leq j \leq q$. If the integrals

$$
J_{a}=\left(H K S_{I}\right) \int_{B} \mathcal{A}\left(f_{1}, \ldots, f_{p}, d g_{1}, \ldots, d g_{j, a}, \ldots, d g_{q}\right)
$$

and

$$
J_{b}=\left(H K S_{I}\right) \int_{B} \mathcal{A}\left(f_{1}, \ldots, f_{p}, d g_{1}, \ldots, d g_{j, b}, \ldots, d g_{q}\right)
$$


exist then the integral

$$
J=\left(H K S_{I}\right) \int_{B} \mathcal{A}\left(f_{1}, \ldots, f_{p}, d g_{1}, \ldots, d g_{j}, \ldots, d g_{q}\right)
$$

also exists and $J=J_{a}+J_{b}$.

c) Let $k$ be a real (or complex) number. If the integral

$$
\left(H K S_{I}\right) \int_{B} \mathcal{A}\left(f_{1}, \ldots, f_{p}, d g_{1}, \ldots, d g_{q}\right)
$$

exists then

$\left.c_{1}\right)$

$$
\begin{aligned}
& \left(H K S_{I}\right) \int_{B} \mathcal{A}\left(f_{1}, \ldots, k f_{i}, \ldots, f_{p}, d g_{1}, \ldots, d g_{q}\right) \\
= & k \cdot\left(H K S_{I}\right) \int_{B} \mathcal{A}\left(f_{1}, \ldots, f_{i}, \ldots, f_{p}, d g_{1}, \ldots, d g_{q}\right)
\end{aligned}
$$

and

$\left.c_{2}\right)$

$$
\begin{aligned}
& \left(H K S_{I}\right) \int_{B} \mathcal{A}\left(f_{1}, \ldots, f_{p}, d g_{1}, \ldots, d\left(k g_{j}\right), \ldots, d g_{q}\right) \\
& =k \cdot\left(H K S_{I}\right) \int_{B} \mathcal{A}\left(f_{1}, v f_{p}, d g_{1}, \ldots, d g_{j}, \ldots, d g_{q}\right) .
\end{aligned}
$$

d) Let

$$
\mathcal{A}_{1}, \mathcal{A}_{2}: X_{1} \times \cdots \times X_{p} \times Y_{1} \cdots \times Y_{q} \rightarrow Z
$$

be bounded multilinear operators and let $k_{1}, k_{2}$ be real (or complex) numbers. If the integrals

$$
\left(H K S_{I}\right) \int_{B} \mathcal{A}_{1}(F, d G) \quad \text { and } \quad\left(H K S_{I}\right) \int_{B} \mathcal{A}_{2}(F, d G)
$$

exist then

$$
\begin{gathered}
\left(H K S_{I}\right) \int_{B}\left(k_{1} \mathcal{A}_{1}+k_{2} \mathcal{A}_{2}\right)(F, d G) \\
=k_{1} \cdot\left(H K S_{I}\right) \int_{B} \mathcal{A}_{1}(F, d G)+k_{2} \cdot\left(H K S_{I}\right) \int_{B} \mathcal{A}_{2}(F, d G) .
\end{gathered}
$$


Proof. We prove only $b$ ). Choose $\epsilon>0$ arbitrarily and let $\delta_{a}$ and $\delta_{b}$ fulfill $D \ll \delta_{a} \Rightarrow\left|S_{I}^{(a)}(D)-J_{a}\right|<\epsilon / 2$ and $D \ll \delta_{b} \Rightarrow\left|S_{I}^{(b)}(D)-J_{b}\right|<\epsilon / 2$ where $S_{I}^{(a)}(D)$ and $S_{I}^{(b)}(D)$ are arbitrary Stieltjes sums belonging to the HKS-integrals with $g_{i, a}$ and $g_{i, b}$ as integrators respectively. Set $\delta=\min \left(\delta_{a}, \delta_{b}\right)$ and let $D$ be any $\delta$-fine partition of $B$. By the multilinearity of $\mathcal{A}$ and the linearity of the Stieltjes quasi volume, see Remark 2.1, we can write

$$
S_{I}(D)=S_{I}^{(a)}(D)+S_{I}^{(b)}(D) .
$$

Now let $J=J_{a}+J_{b}$. We then have

$$
\begin{gathered}
\left.\mid S_{I}(D)-J\right)|=|\left(S_{I}^{(a)}(D)+S_{I}^{(b)}(D)\right)-\left(J_{a}+J_{b}\right) \mid \leq \\
\mid\left(S_{I}^{(a)}(D)-J_{a}|+| S_{I}^{(b)}(D)-J_{b} \mid<\epsilon / 2+\epsilon / 2=\epsilon .\right.
\end{gathered}
$$

This means that the integral

$$
\left(H K S_{I}\right) \int_{B} \mathcal{A}\left(f_{1}, \ldots, f_{p}, d g_{1}, \ldots, d g_{i}, \ldots, d g_{q}\right)
$$

exists and has the value $J$.

Remark 4.3. If we have only one integrator $g_{1}$ then the expression

$$
\mathcal{A}\left[f_{1}\left(s_{1}\right), \ldots, f_{p}\left(s_{p}\right), \sigma_{g_{1}}(C)\right]
$$

preserves the property of interval additivity in the following meaning:

If $\left\{C_{1}, \ldots, C_{m}\right\}$ is a partition of a block $C \subseteq B$ and $s_{1}, \ldots, s_{p} \in C$ then, by the multilinearity of $\mathcal{A}$ and the linearity of the Stieltjes quasi volume, we have:

$$
\begin{gathered}
\mathcal{A}\left[f_{1}\left(s_{1}\right), \ldots, f_{p}\left(s_{p}\right), \sigma_{g_{1}}(C)\right]=\mathcal{A}\left[f_{1}\left(s_{1}\right), \ldots, f_{p}\left(s_{p}\right), \sum_{i=1}^{m} \sigma_{g_{1}}\left(C_{i}\right)\right] \\
=\sum_{i=1}^{m} \mathcal{A}\left[f_{1}\left(s_{1}\right), \ldots, f_{p}\left(s_{p}\right), \sigma_{g_{1}}\left(C_{i}\right)\right] .
\end{gathered}
$$

But, if we have more then one integrator then the expression

$$
\mathcal{A}\left[f_{1}\left(s_{1}\right), \ldots, f_{p}\left(s_{p}\right), \sigma_{g_{1}}(C), \ldots, \sigma_{g_{q}}(C)\right]
$$

is not necessarily interval additive. Consider, for example, the case where we have two integrators (and one integrand) 
$\mathcal{A}\left[f_{1}\left(s_{1}\right), \sigma_{g_{1}}(C), \sigma_{g_{2}}(C)\right]$. Let $\left\{C_{1}, C_{2}\right\}$ be a partition of a block $C \subseteq B$. We have

$$
\begin{gathered}
\mathcal{A}\left[f_{1}\left(s_{1}\right), \sigma_{g_{1}}(C), \sigma_{g_{2}}(C)\right]=\mathcal{A}\left[f_{1}\left(s_{1}\right), \sigma_{g_{1}}\left(C_{1} \cup C_{2}\right), \sigma_{g_{2}}\left(C_{1} \cup C_{2}\right)\right] \\
=\mathcal{A}\left[f_{1}\left(s_{1}\right), \sigma_{g_{1}}\left(C_{1}\right)+\sigma_{g_{1}}\left(C_{2}\right), \sigma_{g_{2}}\left(C_{1}\right)+\sigma_{g_{2}}\left(C_{2}\right)\right] \\
=\mathcal{A}\left[f_{1}\left(s_{1}\right), \sigma_{g_{1}}\left(C_{1}\right), \sigma_{g_{2}}\left(C_{1}\right)\right]+\mathcal{A}\left[f_{1}\left(s_{1}\right), \sigma_{g_{1}}\left(C_{1}\right), \sigma_{g_{2}}\left(C_{2}\right)\right] \\
+\mathcal{A}\left[f_{1}\left(s_{1}\right), \sigma_{g_{1}}\left(C_{2}\right), \sigma_{g_{2}}\left(C_{1}\right)\right]+\mathcal{A}\left[f_{1}\left(s_{1}\right), \sigma_{g_{1}}\left(C_{2}\right), \sigma_{g_{2}}\left(C_{2}\right)\right]
\end{gathered}
$$

Thus, in the general case,

$$
\mathcal{A}\left[f_{1}\left(s_{1}\right), \sigma_{g_{1}}(C), \sigma_{g_{2}}(C)\right]
$$

differs from

$$
\mathcal{A}\left[f_{1}\left(s_{1}\right), \sigma_{g_{1}}\left(C_{1}\right), \sigma_{g_{2}}\left(C_{1}\right)\right]+\mathcal{A}\left[f_{1}\left(s_{1}\right), \sigma_{g_{1}}\left(C_{2}\right), \sigma_{g_{2}}\left(C_{2}\right)\right] .
$$

Therefore, we must include the interval additivity of the expression $\mathcal{A}\left[f_{1}\left(s_{1}\right), \ldots, f_{p}\left(s_{p}\right), \sigma_{g_{1}}(C)\right]$ as an extra assumption, if we need this property to prove a lemma or a theorem (as in Lemma 4.6, and Theorems 4.1 and 5.1).

Lemma 4.6. Let $\Pi=\left\{B_{1}, \ldots, B_{r}\right\}$, be a partition of $B$. Assume that the two following conditions hold:

1. (Interval additivity) If $\left\{C_{1}, \ldots, C_{m}\right\}$ is a partition of a block $C \subseteq B$ and $s_{1}, \ldots, s_{p} \in C$ then

$$
\begin{gathered}
\mathcal{A}\left[f_{1}\left(s_{1}\right), \ldots, f_{p}\left(s_{p}\right), \sigma_{g_{1}}(C), \ldots, \sigma_{g_{q}}(C)\right] \\
=\sum_{i=1}^{m} \mathcal{A}\left[f_{1}\left(s_{1}\right), \ldots, f_{p}\left(s_{p}\right), \sigma_{g_{1}}\left(C_{i}\right), \ldots, \sigma_{g_{q}}\left(C_{i}\right)\right] .
\end{gathered}
$$

2. The integrals $\left(H K S_{I}\right) \int_{B_{k}} \mathcal{A}(F, d G), k=1, \ldots, r$ exist.

Then the integral $\left(H K S_{I}\right) \int_{B} \mathcal{A}(F, d G)$ exists and

$$
\left(H K S_{I}\right) \int_{B} \mathcal{A}(F, d G)=\sum_{k=1}^{r}\left(H K S_{I}\right) \int_{B_{k}} \mathcal{A}(F, d G) .
$$

Proof. For simplicity, we shall prove the case when $B=B_{1} \cup B_{2}$, where $B_{1}$ and $B_{2}$ are two adjacent blocks with one face, $M$, in common. We denote $J_{1}=\left(H K S_{I}\right) \int_{B_{1}} \mathcal{A}(F, d G)$ and $J_{2}=\left(H K S_{I}\right) \int_{B_{2}} \mathcal{A}(F, d G)$. Our aim is to prove that $\left(H K S_{I}\right) \int_{B} \mathcal{A}(F, d G)$ exists and that

$$
\left(H K S_{I}\right) \int_{B} \mathcal{A}(F, d G)=J_{1}+J_{2} .
$$


Given $\epsilon>0$, let $\delta_{1}$ and $\delta_{2}$ be defined on $B_{1}$ and $B_{2}$ such that if the partition $D_{1}$ of $B_{1}$ is $\delta_{1}$-fine, we have $\left|S_{I}^{(1)}\left(D_{1}\right)-J_{1}\right|<\epsilon / 2$ and if the partition $D_{2}$ is $\delta_{2}$-fine we have $\left|S_{I}^{(2)}\left(D_{2}\right)-J_{2}\right|<\epsilon / 2$. Here $S_{I}^{(1)}\left(D_{1}\right)$ and $S_{I}^{(2)}\left(D_{2}\right)$ denote Stieltjes sums for the integrals over $B_{1}$ and $B_{2}$ respectively. Denote by $d(P, M)$ the distance from a point $P$ to the set $M$ and define the gauge

$$
\delta= \begin{cases}\min \left\{\delta_{1}(P), \delta_{2}(P)\right\} & \text { if } P \in M ; \\ \min \left\{\delta_{1}(P), \frac{1}{2} d(P, M)\right\} & \text { if } P \in B_{1} \backslash M ; \\ \min \left\{\delta_{2}(P), \frac{1}{2} d(P, M)\right\} & \text { if } P \in B_{2} \backslash M .\end{cases}
$$

Let $D$ be a $\delta$-fine partition of $B$ consisting of subblocks $C_{k}$ and associated tags. Consider the associated Stieltjes sum $S_{I}(D)$. First we divide all the subblocks into three groups. The first group consists of blocks denoted here by $C_{1, i}$ which are entirely contained in $B_{1}$, the second group consists of blocks denoted by $C_{2, j}$ which are entirely contained in $B_{2}$ and the third group consists of all other subblocks denoted by $C_{3, m}$. Thus, for a subblock $C_{3, m}$, we have $C_{3, m} \cap \operatorname{int}\left(B_{1}\right) \neq \emptyset$ and $C_{3, m} \cap \operatorname{int}\left(B_{2}\right) \neq \emptyset$, where $\operatorname{int}\left(B_{k}\right)$ denotes the interior of $B_{k}$. Due to the definition of $\delta$, all associated tags $\left(s_{1}^{m}, \ldots, s_{p}^{m}\right)$ of $C_{3, m}$ belong to the common face $M$.

We now split each subblock $C_{3, m}$ in into $E_{m}=C_{3, m} \cap B_{1}$ and $F_{m}=C_{3, m} \cap B_{2}$ and associate the same tags $\left(s_{1}^{m}, \ldots, s_{p}^{m}\right)$ to both $E_{m}$ and $F_{m}$. By the interval additivity of $\mathcal{A}$ we have

$$
\begin{aligned}
& \mathcal{A}\left[f_{1}\left(s_{1}^{m}\right), \ldots, f_{p}\left(s_{p}^{m}\right), \sigma_{g_{1}}\left(C_{3, m}\right), \ldots, \sigma_{g_{q}}\left(C_{3, m}\right)\right] \\
& =\mathcal{A}\left[f_{1}\left(s_{1}^{m}\right), \ldots, f_{p}\left(s_{p}^{m}\right), \sigma_{g_{1}}\left(E_{m}\right), \ldots, \sigma_{g_{q}}\left(E_{m}\right)\right] \\
& +\mathcal{A}\left[f_{1}\left(s_{1}^{m}\right), \ldots, f_{p}\left(s_{p}^{m}\right), \sigma_{g_{1}}\left(F_{m}\right), \ldots, \sigma_{g_{q}}\left(F_{m}\right)\right] .
\end{aligned}
$$

Adding the blocks $E_{m}$ to the blocks $C_{1, i}$ (together with associated tags) we get a $\delta_{1}$-fine partition $D^{\prime}$ of $B_{1}$. Similarly, adding the blocks $F_{m}$ to the blocks $C_{2, j}$ we get a $\delta_{2}$-fine partition $D^{\prime \prime}$ of $B_{2}$. Furthermore,

$$
S_{I}(D)=S_{I}\left(D^{\prime}\right)+S_{I}\left(D^{\prime \prime}\right)
$$

so that we have

$$
\left|S_{I}(D)-\left(J_{1}+J_{2}\right)\right| \leq\left|S_{I}\left(D^{\prime}\right)-J_{1}\right|+\left|S_{I}\left(D^{\prime \prime}\right)-J_{2}\right|<\epsilon / 2+\epsilon / 2=\epsilon .
$$

Thus the integral $\left(H K S_{I}\right) \int_{B} \mathcal{A}(F, d G)$ exists and

$$
\left(H K S_{I}\right) \int_{B} \mathcal{A}(F, d G)=J_{1}+J_{2}
$$


Note that in the case when we have only one integrator, the interval additivity condition in Lemma 4.6 is automatically fulfilled (as we mentioned in Remark 4.3).

Definition 4.6. Semivariation. Let $\mathcal{A}: X_{1} \times \cdots \times X_{p} \times Y_{1} \cdots \times Y_{q} \rightarrow Z$ be a multilinear operator. We say that Stieltjes quasi volumes $\sigma_{g_{1}}, \ldots, \sigma_{g_{q}}$ are of bounded semivariation on $B$ with respect to $\mathcal{A}$ if there exists a constant $M_{s}$ such that for any partition $\Pi=\left\{B_{1}, \ldots, B_{r}\right\}$ of $B$ and arbitrary vectors $x_{j}^{i} \in X_{j}$, $i=1, \ldots, r, j=1, \ldots, p$ the following holds

$$
\left|\sum_{i=1}^{r} \mathcal{A}\left[x_{1}^{i}, \ldots, x_{p}^{i}, \sigma_{g_{1}}\left(B_{i}\right), \ldots, \sigma_{g_{q}}\left(B_{i}\right)\right]\right| \leq M_{s} \cdot G_{1} \cdot G_{2} \cdots G_{p} .
$$

where

$$
G_{j}=\max \left\{\left|x_{j}^{i}\right|, i=1, \ldots, n\right\}
$$

Theorem 4.1. Let $X_{1}, \ldots, X_{p}, Y_{1}, \ldots, Y_{q}$ be normed spaces and let $Z$ be a Banach space over the same field. Let $\mathcal{A}: X_{1} \times \cdots \times X_{p} \times Y_{1} \cdots \times Y_{q} \rightarrow Z$ be a multilinear operator. Assume that the following hold:

1. The integrands $f_{j}: B \rightarrow X_{j}$ are continuous functions.

2. Stieltjes quasi volumes $\sigma_{g_{1}}, \ldots, \sigma_{g_{q}}$ are of bounded semivariation.

3. (Interval additivity) If $\left\{C_{1}, \ldots, C_{m}\right\}$ is a partition of a block $C \subseteq B$ and $s_{1}, \ldots, s_{p} \in C$ then

$$
\begin{gathered}
\mathcal{A}\left[f_{1}\left(s_{1}\right), \ldots, f_{p}\left(s_{p}\right), \sigma_{g_{1}}(C), \ldots, \sigma_{g_{q}}(C)\right] \\
=\sum_{i=1}^{m} \mathcal{A}\left[f_{1}\left(s_{1}\right), \ldots, f_{p}\left(s_{p}\right), \sigma_{g_{1}}\left(C_{i}\right), \ldots, \sigma_{g_{q}}\left(C_{i}\right)\right]
\end{gathered}
$$

Then the Riemann-Stieltjes multilinear integral

$$
\left(R S_{I}\right) \int_{B} \mathcal{A}\left(f_{1}, \ldots, f_{p}, d g_{1}, \ldots, d g_{q}\right)
$$

exists.

Proof. To simplify notation we assume that we only have two integrands $f_{1}$ and $f_{2}$. The proof of the general case can be carried out in the same way.

Now we let $\epsilon>0$ have an arbitrary value. Let $M_{1}=\max \left\{\left|f_{1}(s)\right|\right.$, $s \in B)\}$ and $\left.M_{2}=\max \left\{\left|f_{2}(s)\right|, s \in B\right)\right\}$. The functions $f_{1}, f_{2}$ are continuous 
on $B$ and hence they are uniformly continuous on $B$. We can therefore choose $\delta>0$ such that for any partition $\Pi=\left\{B_{k}, k=1, \ldots, r\right\}$ of $B$ with $|\Pi|<\delta$ we have $\left|f_{j}(s)-f_{j}\left(s^{\prime}\right)\right|<\epsilon$ for $j=1,2$ and all $s, s^{\prime} \in B$, if $s$ and $s^{\prime}$ belong to the same subblock in $\Pi$. Let $\Pi^{\prime}=\left\{B_{k, j}\right\}$, where $B_{k}=\cup B_{k, j}$, be any refinement of П. We then have

$$
\begin{gathered}
\left|S_{I}(\Pi)-S_{I}\left(\Pi^{\prime}\right)\right|= \\
\mid \sum_{k} \mathcal{A}\left[f_{1}\left(s_{1}^{k}\right), f_{2}\left(s_{2}^{k}\right), \sigma_{g_{1}}\left(B_{k}\right), \ldots, \sigma_{g_{q}}\left(B_{k}\right)\right] \\
-\sum_{k, j} \mathcal{A}\left[f_{1}\left(s_{1}^{k j}\right), f_{2}\left(s_{2}^{k j}\right), \sigma_{g_{1}}\left(B_{k j}\right), \ldots, \sigma_{g_{q}}\left(B_{k j}\right)\right] \mid .
\end{gathered}
$$

Using the interval additivity of the expression, this can be rewritten as

$$
\begin{gathered}
\mid \sum_{k, j} \mathcal{A}\left[f_{1}\left(s_{1}^{k}\right), f_{2}\left(s_{2}^{k}\right), \sigma_{g_{1}}\left(B_{k j}\right), \ldots, \sigma_{g_{q}}\left(B_{k j}\right)\right] \\
-\sum_{k, j} \mathcal{A}\left[f_{1}\left(s_{1}^{k j}\right), f_{2}\left(s_{2}^{k j}\right), \sigma_{g_{1}}\left(B_{k j}\right), \ldots, \sigma_{g_{q}}\left(B_{k j}\right)\right] \mid \\
=\left|\sum_{k, j} \mathcal{A}\left(f_{1}\left(s_{1}^{k}\right), f_{2}\left(s_{2}^{k}\right), \sigma_{k j}\right)-\mathcal{A}\left(f_{1}\left(s_{1}^{k j}\right), f_{2}\left(s_{2}^{k j}\right), \sigma_{k j}\right)\right|
\end{gathered}
$$

where we simplified notation by writing $\sigma_{k j}$ instead of $\sigma_{g_{1}}\left(B_{k j}\right), \ldots, \sigma_{g_{q}}\left(B_{k j}\right)$. If we here add and subtract $\mathcal{A}\left(f_{1}\left(s_{1}^{k}\right), f_{2}\left(s_{2}^{k j}\right), \sigma_{k j}\right)$ we can use the triangle inequality to estimate 4.4 upwards with $\Sigma_{1}+\Sigma_{2}$ where

$$
\Sigma_{1}=\left|\sum_{k, j} \mathcal{A}\left(f_{1}\left(s_{1}^{k}\right), f_{2}\left(s_{2}^{k}\right), \sigma_{k j}\right)-\mathcal{A}\left(f_{1}\left(s_{1}^{k}\right), f_{2}\left(s_{2}^{k j}\right), \sigma_{k j}\right)\right|
$$

and

$$
\Sigma_{2}=\left|\sum_{k, j} \mathcal{A}\left(f_{1}\left(s_{1}^{k}\right), f_{2}\left(s_{2}^{k j}\right), \sigma_{k j}\right)-\mathcal{A}\left(f_{1}\left(s_{1}^{k j}\right), f_{2}\left(s_{2}^{k j}\right), \sigma_{k j}\right)\right| .
$$

By the multilinearity of $\mathcal{A}$ and the bounded semivariability of the Stieltjes quasi volume we then get

$$
\Sigma_{1}=\left|\sum_{k, j} \mathcal{A}\left(f_{1}\left(s_{1}^{k}\right), f_{2}\left(s_{2}^{k}\right)-f_{2}\left(s_{2}^{k j}\right), \sigma_{k j}\right)\right| \leq M_{s} \cdot M_{1} \cdot \epsilon .
$$

For $\Sigma_{2}$ in the same way to obtain the estimate

$$
\Sigma_{2} \leq M_{s} \cdot \epsilon \cdot M_{2} .
$$


Combining 4.5 and 4.6 we get

$$
\left|S_{I}(\Pi)-S_{I}\left(\Pi^{\prime}\right)\right|<M_{s} \cdot M_{1} \cdot \epsilon+M_{s} \cdot \epsilon \cdot M_{2}=\epsilon M_{s}\left(M_{1}+M_{2}\right)
$$

This proves the existence of $\left(R S_{I}\right) \int_{B} \mathcal{A}\left(f_{1}, f_{2}, d g_{1}, \ldots, d g_{q}\right)$ by Lemma 3.3 . As stated above, an analogous procedure can be employed when dealing with integrals having $p$ integrands.

\section{Moore-Pollard-Stieltjes Multilinear Integrals in $\mathbb{R}^{n}$}

The difference between Moore-Pollard-Stieltjes (MPS) and Riemann-Stieltjes integrals (RS) is the limiting process. The limit of the Stieltjes sums for the MPS-integral is controlled by refinements while the limit for the RS-integral is defined by the norm of partitions.

Definition 5.1. We say that the type I multilinear Moore-Pollard-Stieltjes integral of $f_{i}, i=1, \ldots, p$ with respect to $\mathcal{A}$ and $g_{j}, j=1, \ldots, q$, exists on $B$ if there exists a vector $J_{I} \in Z$ with the following property:

For every $\epsilon>0$, there is a partition $\Pi_{\epsilon}$, such that for any partition $\Pi=$ $\left\{B_{1}, \ldots, B_{r}\right\}$ of the block $B$ that is a refinement of $\Pi_{\epsilon}$, and any choice of points $s_{1}^{i}, \ldots, s_{p}^{i}$ in $B_{i}, i=1, \ldots, r$ we have

$$
\left|J_{I}-S_{I}(\Pi)\right|<\epsilon .
$$

Then we write

$$
J_{I}=\left(M P S_{I}\right) \int_{B} \mathcal{A}\left(f_{1}, \ldots, f_{p}, d g_{1}, \ldots, d g_{q}\right) .
$$

We can also more briefly write

$$
J_{I}=\left(M P S_{I}\right) \int_{B} \mathcal{A}(F, d G)
$$

analogous with earlier notation.

Definition 5.2. By changing type I Stieltjes sums to type II sums in the above definition we get the definition of type II multilinear Moore-PollardStieltjes integral

$$
J_{I I}=\left(M P S_{I I}\right) \int_{B} \mathcal{A}\left(f_{1}, \ldots, f_{p}, d g_{1}, \ldots, d g_{q}\right) .
$$


Remark 5.1. It is obvious that the existence of the type I (type II) RS-integral $R S_{I}$ implies the existence of type I (type II) MPS-integral.

In the next theorem we prove that the existence of the MPS integral, supplied with the interval additivity condition, implies the existence of the HKS integral on the block $B$.

Theorem 5.1. Let $\Pi=\left\{B_{1}, \ldots, B_{r}\right\}$, be a partition of $B$. Assume that the following conditions hold:

1. (Interval additivity) If $\left\{C_{1}, \ldots, C_{m}\right\}$ is a partition of a block $C \subseteq B$ and $s_{1}, \ldots, s_{p} \in C$ then

$$
\begin{gathered}
\mathcal{A}\left[f_{1}\left(s_{1}\right), \ldots, f_{p}\left(s_{p}\right), \sigma_{g_{1}}(C), \ldots, \sigma_{g_{q}}(C)\right] \\
=\sum_{i=1}^{m} \mathcal{A}\left[f_{1}\left(s_{1}\right), \ldots, f_{p}\left(s_{p}\right), \sigma_{g_{1}}\left(C_{i}\right), \ldots, \sigma_{g_{q}}\left(C_{i}\right)\right] .
\end{gathered}
$$

2. The integral $\left(M P S_{I}\right) \int_{B} \mathcal{A}\left(f_{1}, \ldots, f_{p}, d g_{1}, \ldots, d g_{q}\right)$ exists and has the value $J_{I}$.

Then the integral $\left(H K S_{I}\right) \int_{B} \mathcal{A}\left(f_{1}, \ldots, f_{p}, d g_{1}, \ldots, d g_{q}\right)$ exists and has the same value.

Proof. We shall prove this theorem first in the the case when $B$ is a block in the space $\mathbb{R}^{2}$. Fix $\epsilon>0$ and denote $M P S_{I} \int_{B} \mathcal{A}(F, d G)$ by $J_{I}$. Then there exists a partition $\Pi_{\epsilon}$ of $B$ such that for any partition $\Pi$ that is a refinement of $\Pi_{\epsilon}$ we have

$$
\left|S_{I}(\Pi)-J_{I}\right|<\epsilon .
$$

We will now construct a gauge $\delta$ on $B$ to show that the integral $H K S_{I} \int_{B} \mathcal{A}(F, d G)$ also exists with the value $J_{I}$. Let $\left\{B_{1}, \ldots, B_{r}\right\}$ be the partition $\Pi_{\epsilon}$ specified above. We assume further that $\left\{B_{1}, \ldots, B_{r}\right\}$ are non-degenerated intervals. (We can do that since the Stieltjes volume of a degenerated interval is 0 .) Let $M$ be the set of all boundary points and let $V=\left\{v_{1}, \ldots, v_{B}\right\}$ be the set of all different vertices of all the blocks $B_{1}, \ldots, B_{r}$ in $\Pi_{\epsilon}$.

Let $d(P, Q)=\max \left\{\left|p_{k}-q_{k}\right|, k=1, \ldots, n\right\}$ denote the (non-euclidian) distance between points $P=\left(p_{1}, \ldots, p_{n}\right)$ and $Q=$ $\left(q_{1}, \ldots, q_{n}\right)$. Define

$$
\delta_{1}=\min \left\{d\left(v_{k}, v_{l}\right), k \neq l\right\}
$$


Thus, $\delta_{1}$ is the shortest distance between two (different) vertices and $\delta_{1}>0$. Now we define $\delta(P)$ :

$$
\delta(P)= \begin{cases}\delta_{1} / 4, & \text { if } P \in V \\ d(P, V) / 4 & \text { if } P \in M \backslash V \\ d(P, M) / 4 & \text { if } P \in B \backslash M .\end{cases}
$$

Here $d(P, M)=\min \{d(P, X), X \in M\}$ denotes the distance between the point $\mathrm{P}$ and the closed set $M$. Similarly, $d(P, V)$ is the distance between $\mathrm{P}$ and the set $V$.

Let $D_{I}=\left\{\left(\left\{s_{j}^{i}\right\}, C_{i}\right)\right\}$ be a $\delta$-fine partition of $B$. Using blocks $C_{i}$ we construct a new partition that is a refinement of $\Pi_{\epsilon}$. First we divide all the subblocks into two groups. The first group consists of blocks, denoted here by $C_{l}$, which are entirely contained in some $B_{i}$. The second group consists of all other subblocks denoted here by $C_{m}$. Blocks $C_{l}$ (from the first group) we keep unchanged. Blocks $C_{m}$ from the second group (who contain interior points of two or more blocks) we divide in subblocks. From the definition of $\delta$ we have the following properties:

1. If a block $C_{m}$ intersects the set of boundary points $M$ then associated points must be in $M$.

2. Two different vertices can not belong to the same block $C_{m}$.

3. If a vertex $v_{k}$ belongs to $C_{m}$ then all associated points $s_{j}^{m}$ are equal to $v_{k}$, i.e. $s_{j}^{m}=v_{k}, j=1, \ldots, p$.

Let $C_{m}$, with associated points $s_{j}^{m}$, be a block that contains interior points of two or more blocks $B_{i}$. We split $C_{m}$ in subblocks $C_{m, k}$, where $C_{m, k} \subseteq B_{i_{k}}$. The properties 1,2 and 3 show that we can associate the same points $s_{j}^{m}$ to every $C_{m, k}$. For the related term in the Stieltjes sum, using the assumption of interval additivity, we then get

$$
\begin{gathered}
\mathcal{A}\left[f_{1}\left(s_{1}^{m}\right), \ldots, f_{p}\left(s_{p}^{m}\right), \sigma_{g_{1}}\left(C_{m}\right), \ldots, \sigma_{g_{q}}\left(C_{m}\right)\right] \\
=\sum_{k} \mathcal{A}\left[f_{1}\left(s_{1}^{m}\right), \ldots, f_{p}\left(s_{p}^{m}\right), \sigma_{g_{1}}\left(C_{m, k}\right), \ldots, \sigma_{g_{q}}\left(C_{m, k}\right)\right] .
\end{gathered}
$$

Let $D_{I}^{\prime}$ be a partition consisting of the blocks $C_{m, k}$ and those blocks $C_{l}$ lying entirely in some $B_{i}$. Then

$$
S_{I}\left(D_{I}\right)=S_{I}\left(D_{I}^{\prime}\right)
$$


Since $D_{I}^{\prime}$ is a refinement of $\Pi_{\epsilon}$, from $(5.1)$ we have

$$
\left|S_{I}\left(D_{I}^{\prime}\right)-J_{I}\right|<\epsilon
$$

and therefore

$$
\left|S_{I}\left(D_{I}\right)-J_{I}\right|<\epsilon
$$

This means that $\left(H K S_{I}\right) \int_{B} \mathcal{A}\left(f_{1}, \ldots, f_{p}, d g_{1}, \ldots, d g_{q}\right)$ exists and has the value $J_{I}$, which completes the proof in $\mathbb{R}^{2}$.

Defining $\delta$ in a similar way we can prove this theorem in the space $\mathbb{R}^{n}$. For example, in the space $\mathbb{R}^{3}$ we define

$$
\delta(P)= \begin{cases}\delta_{1} / 4, & \text { if } P \in V \\ d(P, V) / 4 & \text { if } P \in M_{1} \backslash V \\ d\left(P, M_{1}\right) / 4 & \text { if } P \in M_{2} \backslash V \\ d\left(P, M_{2}\right) / 4 & \text { if } P \in B \backslash M_{2}\end{cases}
$$

where $V$ denotes all different vertices, $M_{1}$ points on edges and $M_{2}$ boundary points for all blocks $\left\{B_{1}, \ldots, B_{r}\right\}$ of the partition $\Pi_{\epsilon}$. Then we repeat the same procedure as in the case of the space $\mathbb{R}^{2}$.

\section{References}

[1] R. G. Bartle, A Modern theory of integration, American Mathematical Society, (2001).

[2] A. Halilović, Two types of multilinear Stieltjes integrals in the HenstockKurzweil sense, Sarajevo Journal of Mathematics, Vol.5, 17 (2009), 21-39.

[3] A. Halilović, On the existence of the Henstock-Kurzweil-Stieltjes multilinear Integral, International Journal of Pure and Applied Mathematics IJPAM, 55 , No.4 (2009), 581-594

[4] A. Halilović, Semi-variation and multilinear Stieltjes integrals, Glasnik Matematički, III 32(52) (1997), 17-28.

[5] R. Henstock, Lectures on the theory of integration, World Scientific Publishing, Singapore 1988.

[6] P. Y. Lee, Lanzhou lectures on Henstock integration, Word Scientific Publ.Co., Singapore-New Jersey-London-Hong Kong, 1989. 
[7] G. E. Shilov, B. L. Gurevich, Integral measure and derivative: a unified approach, Prentice Hall, Inc., 1966. 